Fighlighted Paper selected by Bditor-in-Ghief

\title{
Scavenging of Hydroxyl Radicals in Aqueous Solution by Astaxanthin Encapsulated in Liposomes
}

\author{
Susumu Hama, ${ }^{a}$ Sachiko Uenishi, ${ }^{a}$ Asako Yamada, ${ }^{a}$ Takashi Ohgita, ${ }^{a}$ Hiroyuki Tsuchiya, ${ }^{a}$ \\ Eiji Yamashita, ${ }^{b}$ and Kentaro Kogure*,a \\ ${ }^{a}$ Department of Biophysical Chemistry, Kyoto Pharmaceutical University; Misasagi-Nakauchicho 5, Yamashina-ku, \\ Kyoto 607-8414, Japan: and ${ }^{b}$ Fuji Chemical Industry Co., Ltd.; 55 Yokohoonji, Kamiichi-machi, Nakaniikawa-gun, \\ Toyama 930-0397, Japan. Received August 15, 2012; accepted September 12, 2012
}

Astaxanthin (Asx) is known to be a potent quencher of singlet oxygen and an efficient scavenger of superoxide anion. Therefore, Asx would be expected to be a useful antioxidant for the prevention of oxidative stress, a causative factor in severe diseases such as ischemic reperfusion injury. However, it is still unclear whether Asx has scavenging capability against the most potent reactive oxygen species (ROS), hydroxyl radical, because the hydrophobicity of Asx prevents analysis of hydroxyl radical scavenging ability in aqueous solution. In this study, to solve this problem, liposomes containing Asx (Asx-lipo), which could be dispersed in water, were prepared, and the scavenging ability of Asx-lipo for the hydroxyl radical was examined. The liposomal formulation enabled encapsulation of a high concentration of Asx. Asx-lipo gave a dose-dependent reduction of chemiluminescence intensity induced by hydroxyl radical in aqueous solution. Hydroxyl radical scavenging of Asx was more potent than $\alpha$-tocopherol. The absorbance of Asx in the liposome decreased after reduction of hydroxyl radicals, indicating the direct hydroxyl radical scavenging by Asx. Moreover, Asx-lipo prevented hydroxyl radical-induced cytotoxicity in cultured NIH-3T3 cells. In conclusion, Asx has potent scavenging capability against hydroxyl radicals in aqueous solution, and this paper is the first report regarding hydroxyl radial scavenging by Asx.

Key words astaxanthin; liposome; hydroxyl radical; radical scavenging

Astaxanthin (Asx, 3,3'-dihydroxy- $\beta, \beta$-carotene-4,4'-dione; Fig. 1 chemical structure) is a common pigment found in algae, fish, and birds. ${ }^{1,2)}$ Asx has been reported to be more effective than a representative carotenoid, $\beta$-carotene, for prevention of singlet oxygen, as well as lipid peroxidation in biological membranes. ${ }^{3-7)}$ The direct radical scavenging capability of Asx has been confirmed using the synthetic radical 1,1-diphenyl-2-picrylhydrazyl. ${ }^{8)}$ Previously, we suggested that the potent antioxidation activity of astaxanthin in biomembranes could be attributable to the conjugated polyene and terminal ring moieties of Asx that trap radicals on the membrane surface and within the membrane, respectively. ${ }^{6)}$ Moreover, it has been reported that some Asx derivatives could scavenge superoxide anion radical. ${ }^{9,10)}$ Highly active reactive oxygen species (ROS), such as superoxide anion radical and hydroxyl radical, have been considered causative agents of severe diseases, such as arteriosclerosis and ischemic reperfusion injury. ${ }^{11-13)}$ Therefore, Asx would be expected to prevent the pathogenesis of such ROS-related diseases by scavenging highly active ROS. However, there have been no reports regarding the capability of Asx to scavenge hydroxyl radical, although it was reported that Asx inhibited intracellular radical generation in cultured cells induced by treatment with Fenton's reagent. ${ }^{14)}$ Since the hydrophobicity of Asx prevents its analysis in aqueous solution, determining the capacity of Asx to react with hydroxyl radical in aqueous solution would be aided by a liposomal formulation that would allow homogeneous dispersion of the poorly water soluble Asx. Previously, we suggested that Asx penetrates through the membrane of liposomes, and the terminal rings of Asx interact with polar head groups of membrane lipids by hydrogen bonding. ${ }^{6)}$ With the terminal ring moieties of Asx located at the membrane

The authors declare no conflict of interest. surface, they would be available to react with exogenous free radicals in the aqueous phase. ${ }^{6}$ Therefore, liposome encapsulated Asx (Asx-lipo) was expected to be an ideal formulation for measurement of Asx hydroxyl radical scavenging ability in aqueous solution.

In this study, Asx-lipo was prepared, and its hydroxyl radical scavenging activity in aqueous solution was examined and compared with the activity of the well known antioxidants $\beta$-carotene and $\alpha$-tocopherol also in liposome formulations. Moreover, the protective effect of Asx-lipo on hydroxyl radical-induced cytotoxicity was examined. This report constitutes the first study of the hydroxyl radical scavenging capability of Asx in aqueous solution.

\section{MATERIALS AND METHODS}

Materials Astaxanthin, $\beta$-carotene, and $\alpha$-tocopherol were<smiles>[B]OC(=O)OC(C)CCCC(C)CCCC1(C)CCc2c(C)c(O)c(C)c(C)c2O1</smiles>

Fig. 1. Chemical Structures of Astaxanthin (Asx), $\beta$-Carotene ( $\beta$-Caro) and $\alpha$-Tocophero $(\alpha$-Toco) 
purchased from Sigma-Aldrich Co., LLC. (St. Louis, MO, U.S.A.). Egg phosphatidylcholine (EPC) and dimyristoyl phosphatidylcholine (DMPC) were obtained from NOF Corporation (Tokyo, Japan). Luminol reagent was purchased from Nacalai Tesque (Kyoto, Japan). NIH3T3 cells were obtained from RIKEN BRC CellBank (Wako, Japan). All other reagents were of the highest grade commercially available.

Preparation of Liposomal Formulations of the Antioxidants Astaxanthin, $\boldsymbol{\beta}$-Carotene, and $\boldsymbol{\alpha}$-Tocopherol EPC liposome and DMPC liposome encapsulated antioxidant formulations were prepared using a lipid hydration method. Chloroform solutions containing $1 \mu \mathrm{mol}$ of EPC or DMPC, and various amounts of the antioxidants Asx, $\beta$-carotene, or $\alpha$-tocopherol were dried to a thin film under vacuum using a rotary evaporator (N-1000, EYELA, Tokyo, Japan). The dried lipid film was hydrated with $0.1 \mathrm{~mL}$ of $10 \mathrm{~mm}$ Tris buffer $(\mathrm{pH}$ 7.4) at room temperature to obtain a liposomal suspension, and the particles were diminished in size by sonication using a bath-type sonicator (AU-25C, Aiwa, Tokyo, Japan). The diameter of liposome encapsulated antioxidant was measured by the dynamic light scattering method using a Zetasizer nano (Malvern Instruments Ltd., U.K.).

Measurement of Chemiluminescence Intensity Dependent on Hydroxyl Radical Production by Fenton Reaction Hydroxyl radical generation was evaluated by chemiluminescence intensity according to the previously reported method. ${ }^{15)}$ To generate hydroxyl radicals, $0.1 \mathrm{~mL}$ of $0.25 \mathrm{M} \mathrm{H}_{2} \mathrm{O}_{2}$ (final concentration: $48 \mathrm{~mm}$ ), $50 \mu \mathrm{L}$ of $10 \mathrm{~mm}$ liposomal suspension (final lipid concentration: $0.96 \mathrm{~mm}$ ), $0.17 \mathrm{~mL}$ of $1 \mathrm{~mm}$ luminol solution (final concentration: $330 \mu \mathrm{M}$ ), and $0.2 \mathrm{~mL}$ of $1 \mathrm{~mm}$ $\mathrm{FeSO}_{4}$ solution (final concentration: $385 \mu \mathrm{M}$ ) were mixed in a test tube. Hydroxyl radicals are known to be generated by Fenton reaction ${ }^{16)}$ as follows; $\mathrm{Fe}^{2+}+\mathrm{H}_{2} \mathrm{O}_{2} \rightarrow \mathrm{Fe}^{3+}+\mathrm{OH}^{-}+\cdot \mathrm{OH}$.

Therefore, the generated amount of hydroxyl radicals might be $385 \mu \mathrm{M}$ in theory. Then, $5 \mathrm{~s}$ after mixing, chemiluminescence intensity was measured for $2 \mathrm{~min}$ by a LuminescencerPSN (ATTO, Tokyo, Japan).

Measurement of Absorption Spectra of Astaxanthin in Liposomes before and after Incubation with Fenton's Reagent Absorption spectra of Asx-lipo suspension before and after incubation with $\mathrm{FeSO}_{4}$ and $\mathrm{H}_{2} \mathrm{O}_{2}$ were measured by microplate-reader infiniteM200 (Tecan Group Ltd., Swiss). The concentrations of liposomal suspension, $\mathrm{FeSO}_{4}$ solution, and $\mathrm{H}_{2} \mathrm{O}_{2}$ were the same as described above. Chemiluminescence intensity was measured $5 \mathrm{~s}$ after mixing for $2 \mathrm{~min}$ by a Luminescencer-PSN. The absorption spectrum of EPC liposomes without astaxanthin was subtracted from each absorption spectrum of Asx-lipo to cancel liposomal turbidity. Therefore, the absorption spectra presented in Fig. 4 are differential spectra between liposomes with and without astaxanthin.
Estimation of Cytotoxicity by Hydroxyl Radical Produced by Fenton Reaction NIH3T3 cells were cultured in Dulbecco's modified Eagle's medium (DMEM) containing $10 \%$ fetal bovine serum (FBS) at $37^{\circ} \mathrm{C}$ and in a $5 \% \mathrm{CO}_{2}$ atmosphere for $24 \mathrm{~h}$. The medium was removed, and then DMEM containing $1 \mathrm{~mm}$ of liposomes was added to the cells. The cells were incubated with the medium containing liposomes for $30 \mathrm{~min}$ at $37^{\circ} \mathrm{C}$. After incubation, the medium was removed, and the cells were incubated with DMEM containing $10 \mathrm{mM}$ $\mathrm{FeSO}_{4}$ and $10 \mathrm{~mm} \mathrm{H}_{2} \mathrm{O}_{2}$ at $37^{\circ} \mathrm{C}$ for $30 \mathrm{~min}$. After incubation, DMEM was removed, and the cells were collected by treatment with trypsin. The collected cells were stained with $0.4 \%$ trypan blue, and the numbers of total cells and stained cells were counted.

Statistical Analysis Statistical significance was determined using Student's $t$-test. $p$ values $<0.05$ were considered to be significant.

\section{RESULTS AND DISCUSSION}

Preparation of liposomes containing astaxanthin, $\boldsymbol{\beta}$-carotene, and $\boldsymbol{\alpha}$-tocopherol In this study, we chose EPC, which is used widely for liposome formulation, as a base lipid of liposomes to encapsulate Asx. Liposomes were prepared by the conventional lipid film hydration method. The average diameter of EPC liposomes without Asx (EPC-lipo) was approximately $150 \mathrm{~nm}$, although the values widely varied (Table 1). EPC-lipo encapsulating Asx (Asx-EPC-lipo) were prepared by the same method as for EPC-liposomes. The average diameter of EPC-lipo containing various amounts of Asx showed a tendency to increase with increasing amounts of Asx (Table 1). For comparison with Asx-lipo, EPC-lipo containing $\beta$-carotene ( $\beta$-caro-EPC-lipo) and $\alpha$-tocopherol ( $\alpha$-toco-EPC-lipo) were prepared. The average size of $\beta$-caro-EPC-lipo also increased with an increase in $\beta$-carotene content; however, the diameter of $\alpha$-toco-EPC-lipo was not dependent on the amount of $\alpha$-tocopherol (Table 1). These results suggest that the increase in diameter from EPC-lipo was due to Asx and $\beta$-carotene intercalation into the lipid membrane via the polyene portion of the structures. The effect of incorporating $\alpha$-tocopherol on liposomal size differed from the carotenoids presumably because its smaller size and amphiphilic structure would more readily maintain the liposome size (Fig. 1), although the hydroxyl group of the chroman ring has also been suggested to locate around the hydrophobic/hydrophilic interface of lipid membranes similar to astaxanthin. ${ }^{17)}$

Effect of Astaxanthin Liposomes on Chemiluminescence Intensity Depending on Generation of Hydroxyl Radical by the Fenton Reaction In order to evaluate the scavenging ability of Asx for hydroxyl radicals, we examined the effect

Table 1. Average Diameters of EPC Liposomes (EPC-Lipo) and DMPC Liposomes (DMPC-Lipo) Encapsulating Various amounts of Asx, $\beta$-Caro and $\alpha$-Toco

\begin{tabular}{cccccc}
\hline \hline $\begin{array}{c}\text { Amount of antioxidant } \\
\text { (mol\%) }\end{array}$ & 0 & 1 & 2 & 4 & 8 \\
\hline Asx-EPC-lipo & $151.4 \pm 125.0$ & $170.1 \pm 119.4$ & $168.1 \pm 133.1$ & $198.6 \pm 140.7$ & $333.6 \pm 80.2$ \\
$\beta$-Caro-EPC-lipo & $151.4 \pm 125.0$ & $166.3 \pm 80.2$ & $203.8 \pm 56.7$ & $213.8 \pm 99.2$ & $216.3 \pm 92.4$ \\
$\alpha$-Toco-EPC-lipo & $151.4 \pm 125.0$ & $114.1 \pm 51.0$ & $95.3 \pm 28.5$ & $108.9 \pm 31.1$ & $188.6 \pm 232.4$ \\
Asx-DMPC-lipo & $112.5 \pm 51.2$ & - & - & $232.9 \pm 268.9$ & $171.8 \pm 50.7$ \\
\hline
\end{tabular}

The average diameter of liposomes was measured by dynamic light scattering. Data shown are the means with S.D. from multiple measurements ( $n>5)$. 


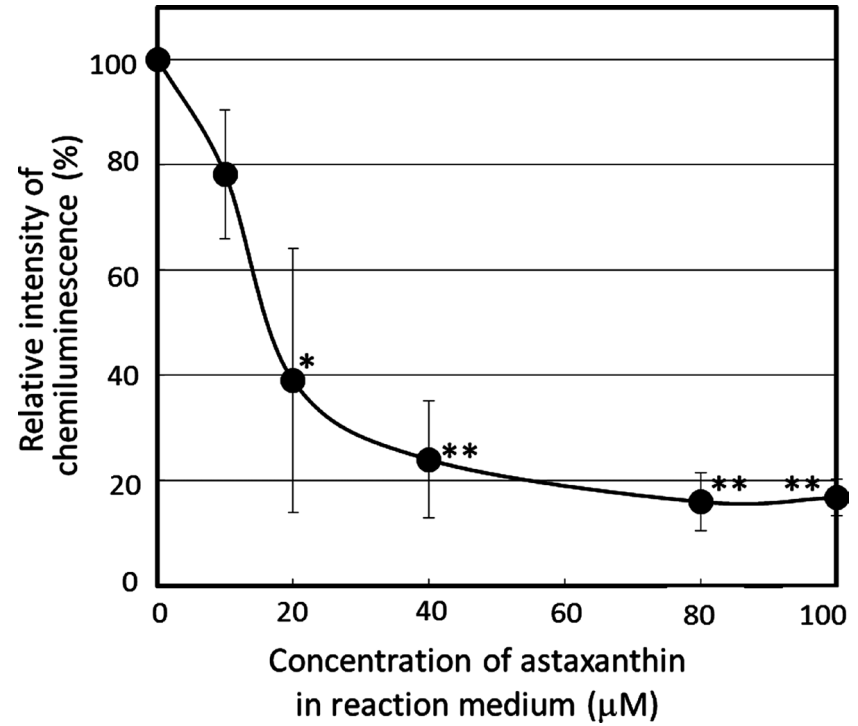

Fig. 2. Effect of Asx-EPC-Lipo on Chemiluminescence Intensity Induced by Hydroxyl Radical Production

Chemiluminescence intensity of each concentration of Asx in Asx-EPC-lipo suspension represented as a relative percentage against that of control EPC-lipo. $\mathrm{H}_{2} \mathrm{O}_{2}$, MCLA, and $\mathrm{FeSO}_{4}$ solution were added to the Asx-EPC-lipo suspension, then the chemiluminescence intensity was measured by a luminometer $5 \mathrm{~s}$ after the addition of Fenton's reagent. Data are shown as means with S.D. from separate measurements $(n>3)$. Statistical significance against control EPC-lipo without Asx is represented as $* p<0.05$ or $* * p<0.01$.

of EPC-lipo encapsulation of various amounts of Asx on the chemiluminescence intensity that depended on hydroxyl radical generation by the Fenton reaction in aqueous solution. As shown in Fig. 2, the chemiluminescence intensity decreased in a dose-dependent manner with respect to Asx in EPC-lipo. In particular, relatively low concentrations $(<20 \mu \mathrm{M})$ of Asx significantly reduced the chemiluminescence intensity. This result indicates for the first time that Asx encapsulated in EPC-lipo membranes can scavenge hydroxyl radicals in aqueous solution. As suggested previously, the terminal rings of Asx would be located at the membrane surface, and the polyene moiety would be embedded in the hydrophobic region of the lipid membrane ${ }^{6}$; therefore, the terminal rings of Asx on the liposome surface would be available to scavenge hydroxyl radicals.

$\beta$-Caro-EPC-lipo and $\alpha$-toco-EPC-lipo also reduced the hydroxyl radical dependent chemiluminescence intensity. It has been reported that $\alpha$-tocopherol in liposomal membranes and micelle structures can scavenge hydroxyl radicals generated in aqueous solution. ${ }^{18)}$ Recently, direct observation of $\beta$-carotene reaction with hydroxyl radical was confirmed using a photoFenton reagent to generate hydroxyl radicals in acetonitrile/ tetrahydrofuran solution, ${ }^{19)}$ although similar measurements have not been performed in aqueous solution. Therefore, this is the first report regarding the hydroxyl radical scavenging reactions of carotenoids including $\beta$-carotene and Asx in aqueous solution. The concentrations of antioxidants encapsulated in EPC-lipo producing 50\% inhibition of chemiluminescence intensity $\left(\mathrm{IC}_{50}\right)$ are summarized in Table 2. The $\mathrm{IC}_{50} \mathrm{~s}$ of $\beta$-caro-EPC-lipo and $\alpha$-toco-EPC-lipo were 20 and $40 \mu \mathrm{M}$, respectively. The $\mathrm{IC}_{50}$ value of Asx-EPC-lipo was lower than that of $\alpha$-toco-EPC-lipo, which indicates Asx encapsulated in EPC-lipo is a more potent scavenger of hydroxyl radicals generated in aqueous solution than the liposome formulation
Table 2. $\mathrm{IC}_{50}$ Values of Hydroxyl Radical Scavenging by EPC-Lipo Encapsulated Asx, $\beta$-Caro, and $\alpha$-Toco

\begin{tabular}{cc}
\hline \hline Antioxidant & $\mathrm{IC}_{50}(\mu \mathrm{M})$ \\
\hline Astaxanthin & 18 \\
$\beta$-Carotene & 20 \\
$\alpha$-Tocopherol & 40 \\
\hline
\end{tabular}

$\mathrm{IC}_{50}$ values were obtained from the dose-dependent decrease by EPC-lipo encapsulated Asx, $\beta$-caro, and $\alpha$-toco on chemiluminescence intensity induced by hydroxyl radical production.

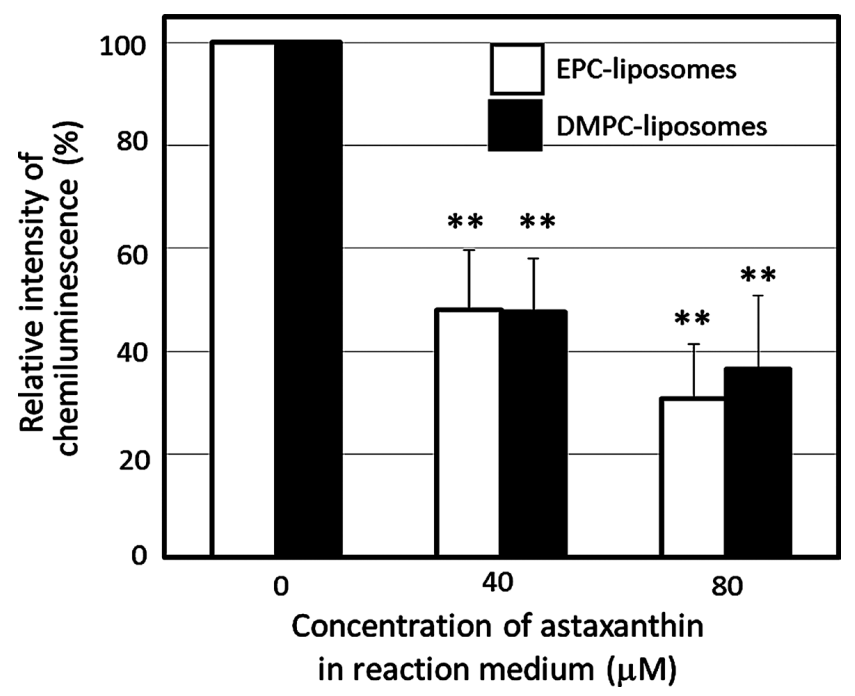

Fig. 3. Effect of Asx Encapsulated in EPC-Lipo Consisting of Unsaturated Acyl Chain and DMPC-Lipo Consisting of Saturated Acyl Chains on Hydroxyl Radical Dependent Chemiluminescence Intensity

Chemiluminescence intensity for each concentration of Asx in EPC-lipo suspension (open column) and DMPC-lipo (closed column) are represented as relative percentages against that of control EPC-lipo. Data are shown as means with S.D. from separate measurements $(n>3)$. Statistical significance against control EPC-lipo or DMPC-lipo without Asx is represented as $* * p<0.01$.

of $\alpha$-tocopherol. The potent hydroxyl radical scavenging activity of Asx may be due to dual radical trapping by the terminal ring as well as the conjugated polyene moieties of Asx on the membrane surface and within the membrane. $\left.{ }^{6}\right)$ On the other hand, the radical trapping site of $\alpha$-tocopherol is limited to a phenolic group, although $\alpha$-tocopherol diffusing freely in the lipid bilayer would effectively react with hydroxyl radicals generated on the outer layer surface of liposomes because of the location of $\alpha$-tocopherol is not fixed in the lipid membranes. $^{20)}$ In this experimental condition, luminol molecules would localize at the membrane interface due to its hydrophobicity. Therefore, Asx might competitively scavenge hydroxyl radicals with luminol at the interface of lipid membranes.

Effect of Unsaturated Acyl Chains in EPC on Hydroxyl Radical Scavenging of Asx-Lipo Since EPC consists of unsaturated fatty acids, which can react with ROS, we were interested in ascertaining whether the unsaturated acyl chains of EPC were responsible for the preventative effect of Asx-EPClipo on the hydroxyl radical dependent chemiluminescence intensity. In order to confirm the participation of unsaturated acyl chains in hydroxyl radical scavenging by Asx-EPC-lipo, DMPC, which consists of saturated fatty acids, was used as a control base lipid for liposome encapsulated Asx. The size of DMPC liposomes without Asx (DMPC-lipo) also increased slightly with an increase in the amount of Asx, although the 
average diameter of DMPC-lipo was less than that of EPC-lipo (Table 1). As shown in Fig. 3, DMPC liposomes encapsulating Asx (Asx-DMPC-lipo) also decreased the hydroxyl radical dependent chemiluminescence intensity in a dose-dependent manner. The Asx-DMPC-lipo had almost the same scavenging effect on hydroxyl radicals as Asx-EPC-lipo. In addition, chemiluminescence intensity of DMPC-lipo without Asx was also the same as that of EPC-lipo without Asx (Supplemental Fig. 1). These results indicate that the unsaturated acyl chains of EPC did not participate in the scavenging capability of Asx-EPC-lipo and that Asx itself in liposomal membranes is responsible for hydroxyl radical scavenging.

Evaluation of Direct Reaction of Asx in Liposomes with Hydroxyl Radical Recently, the direct reaction of $\beta$-carotene with hydroxyl radicals was evaluated from the decrease of characteristic peaks in visible and near-infrared absorption spectra. ${ }^{17)}$ To obtain evidence regarding direct scavenging of hydroxyl radical by Asx, absorption spectra of Asx-lipo before and after the addition of Fenton's reagent were measured (Fig. 4). Asx-lipo gave an absorption peak at $470 \mathrm{~nm}$, which was almost the same wave length as reported previously for Asx in acetonitrile/tetrahydrofuran solution, ${ }^{19)}$ which was dependent on the amount of Asx encapsulated within the liposomes. Upon addition of Fenton's reagents into the suspension, peak absorbance values for Asx at both $40 \mu \mathrm{M}$ and $80 \mu \mathrm{M}$ concentrations decreased as shown in Fig. 4. These results indicate that the conjugated polyene chain moiety was disrupted by hydroxyl radicals and suggests that Asx in liposomes scavenged hydroxyl radicals by direct reaction with its polyene moiety. Asx might react with hydroxyl radicals at the interface of lipid membranes, since hydroxyl radicals would be generated by $\mathrm{Fe}^{2+} / \mathrm{H}_{2} \mathrm{O}_{2}$ accumulated on the surface of liposomal membranes. The decrease in the absorbance of $80 \mu \mathrm{M}$ Asx was almost double that of the $40 \mu \mathrm{M}$ concentration, although the difference in chemiluminescence intensity at $40 \mu \mathrm{M}$ and $80 \mu \mathrm{M}$ of Asx was not significant, i.e., the absorbance change of Asx did not correspond to hydroxyl radical scavenging shown in Figs. 2 and 3. Therefore, $40 \mu \mathrm{M}$ Asx would be enough to scavenge hydroxyl radicals in this condition. Higher decrease in the absorbance of $80 \mu \mathrm{M} \mathrm{Asx}$ than that of $40 \mu \mathrm{M}$ Asx might be due to reaction of surplus Asx with Asx radicals generated by hydroxyl radical scavenging.

Effect of Asx-EPC-Lipo on Cytotoxicity Induced by Hydroxyl Radical Since Asx-EPC-lipo showed a significant scavenging effect on hydroxyl radicals in aqueous solution, Asx-EPC-lipo was also expected to protect cells from hydroxyl radical induced cytotoxicity. Previously, we reported that topical administration of Asx-EPC-lipo protected skin from UV-induced damages. ${ }^{21)}$ Thus, we examined the protective effect of Asx-EPC-lipo on hydroxyl radical-induced cytotoxicity in the cultured mouse skin fibroblast cell line, NIH3T3 cells. As shown in Fig. 5, Asx-EPC-lipo prevented hydroxyl radicalinduced cytotoxicity in a dose-dependent manner. Since Asxlipo would be internalized into cells via endocytosis after a $30 \mathrm{~min}$ incubation and removal of non-internalized free liposomes, the preventative effect was probably due to radical scavenging on the cell surface by Asx distributed in the cell plasma membranes.

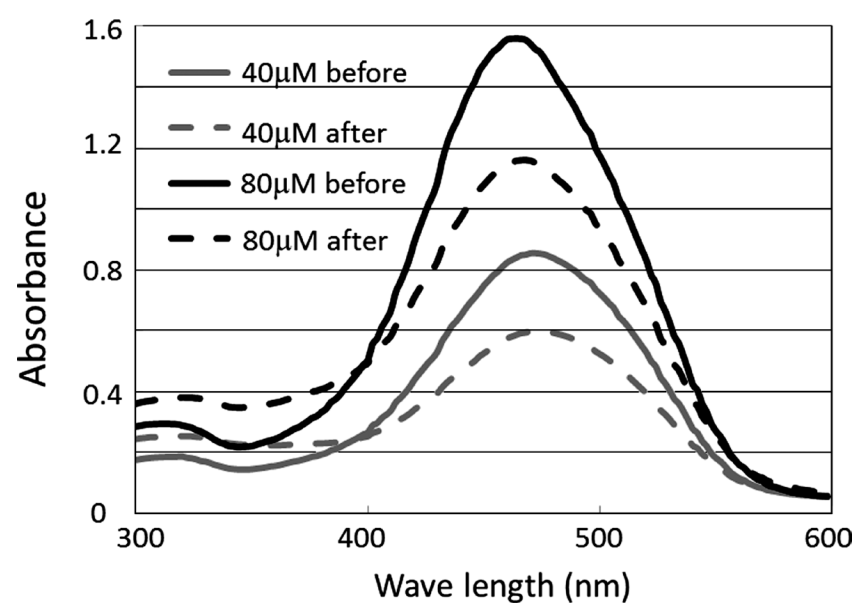

Fig. 4. Absorption Spectra of Asx-Lipo before and after Addition of Fenton's Reagent

Absorption spectrum of liposome suspension with $40 \mu \mathrm{M}$ and $80 \mu \mathrm{M}$ of Asx was measured at visible wavelengths ranging between 300 to $600 \mathrm{~nm}$. Black and gray lines are absorption spectra of $80 \mu \mathrm{M}$ and $40 \mu \mathrm{M}$ Asx, respectively, and solid and dotted lines represent before and immediately after addition of Fenton's reagent, respectively.

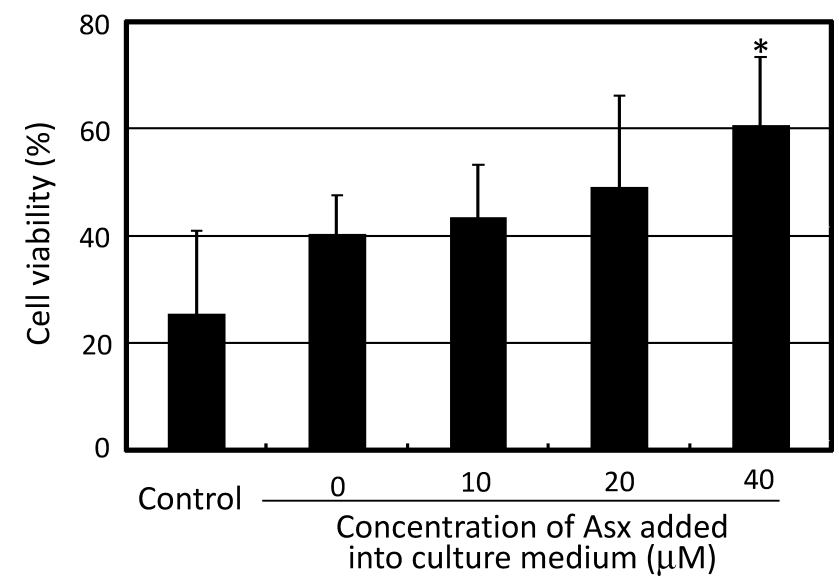

Fig. 5. Effects of EPC-Lipo Encapsulated Asx on the Viability of Cells Treated with Fenton's Reagent

$\mathrm{H}_{2} \mathrm{O}_{2}$ and $\mathrm{FeSO}_{4}$ solution were added to NIH3T3 cells after incubation with liposome suspension. Cell viability was evaluated by staining with trypan blue. Data shown are means with S.D. from separate measurements $(n>3)$. Statistical significance against control is represented as $* p<0.05$.

\section{CONCLUSION}

In this study, Asx-EPC-lipo was prepared, and its capability to scavenge hydroxyl radical in aqueous solution was examined. Asx-EPC-lipo demonstrated significant hydroxyl radical scavenging activity in aqueous solution, which was more potent than either EPC-lipo encapsulated $\beta$-carotene or $\alpha$-tocopherol. The absorption spectrum of Asx in liposomes was reduced in the presence of hydroxyl radical, which indicates that Asx reacted directly with hydroxyl radical. Moreover, Asx-EPC-lipo prevented cell cytotoxicity induced by hydroxyl radical generated in an aqueous phase. This is the first report regarding the hydroxyl radical scavenging capability of Asx in aqueous solution. 


\section{REFERENCES}

1) Guerin M, Huntley ME, Olaizola M. Haematococcus astaxanthin: applications for human health and nutrition. Trends Biotechnol., 21, 210-216 (2003)

2) Maoka T. Carotenoids in marine animals. Mar. Drugs, 9, 278-293 (2011).

3) Terao J. Antioxidant activity of beta-carotene-related carotenoids in solution. Lipids, 24, 659-661 (1989).

4) Lim BP, Nagao A, Terao J, Tanaka K, Suzuki T, Takama K. Antioxidant activity of xanthophylls on peroxyl radical-mediated phospholipid peroxidation. Biochim. Biophys. Acta, 1126, 178-184 (1992).

5) Palozza P, Krinsky NI. Astaxanthin and canthaxanthin are potent antioxidants in a membrane model. Arch. Biochem. Biophys., 297, 291-295 (1992).

6) Goto S, Kogure K, Abe K, Kimata Y, Kitahama K, Yamashita E, Terada $H$. Efficient radical trapping at the surface and inside the phospholipid membrane is responsible for highly potent antiperoxidative activity of the carotenoid astaxanthin. Biochim. Biophys. Acta, 1512, 251-258 (2001).

7) Stahl W, Sies H. Bioactivity and protective effects of natural carotenoids. Biochim. Biophys. Acta, 1740, 101-107 (2005).

8) Kamath BS, Srikanta BM, Dharmesh SM, Sarada R, Ravishankar GA. Ulcer preventive and antioxidative properties of astaxanthin from Haematococcus pluvialis. Eur. J. Pharmacol., 590, 387-395 (2008).

9) Cardounel AJ, Dumitrescu C, Zweier JL, Lockwood SF. Direct superoxide anion scavenging by a disodium disuccinate astaxanthin derivative: Relative efficacy of individual stereoisomers versus the statistical mixture of stereoisomers by electron paramagnetic resonance imaging. Biochem. Biophys. Res. Commun., 307, 704-712 (2003).

10) Jackson HL, Cardounel AJ, Zweier JL, Lockwood SF. Synthesis, characterization, and direct aqueous superoxide anion scavenging of a highly water-dispersible astaxanthin-amino acid conjugate.
Bioorg. Med. Chem. Lett., 14, 3985-3991 (2004).

11) Cathcart MK. Regulation of superoxide anion production by NADPH oxidase in monocytes/macrophages: contributions to atherosclerosis. Arterioscler. Thromb. Vasc. Biol., 24, 23-28 (2004).

12) Kruszewski M. The role of labile iron pool in cardiovascular diseases. Acta Biochim. Pol., 51, 471-480 (2004).

13) Warner DS, Sheng H, Batinić-Haberle I. Oxidants, antioxidants and the ischemic brain. J. Exp. Biol., 207, 3221-3231 (2004).

14) Nakajima $Y$, Inokuchi $Y$, Shimazawa $M$, Otsubo $K$, Ishibashi $T$, Hara H. Astaxanthin, a dietary carotenoid, protects retinal cells against oxidative stress in-vitro and in mice in-vivo. J. Pharm. Pharmacol., 60, 1365-1374 (2008).

15) Hirayama O, Yida M. Evaluation of hydroxyl radical-scavenging ability by chemiluminescence. Anal. Biochem., 251, 297-299 (1997).

16) Jomova K, Valko M. Advances in metal-induced oxidative stress and human disease. Toxicology, 283, 65-87 (2011).

17) Atkinson J, Harroun T, Wassall SR, Stillwell W, Katsaras J. The location and behavior of alpha-tocopherol in membranes. Mol. Nutr. Food Res., 54, 641-651 (2010).

18) Fukuzawa K, Gebicki JM. Oxidation of alpha-tocopherol in micelles and liposomes by the hydroxyl, perhydroxyl, and superoxide free radicals. Arch. Biochem. Biophys., 226, 242-251 (1983).

19) Chen $\mathrm{CH}$, Han RM, Liang R, Fu LM, Wang P, Ai XC, Zhang JP, Skibsted LH. Direct observation of the $\beta$-carotene reaction with hydroxyl radical. J. Phys. Chem. B, 115, 2082-2089 (2011).

20) Fukuzawa K, Ikebata W, Shibata A, Kumadaki I, Sakanaka T, Urano S. Location and dynamics of alpha-tocopherol in model phospholipid membranes with different charges. Chem. Phys. Lipids, 63, 69-75 (1992).

21) Hama S, Takahashi K, Inai Y, Shiota K, Sakamoto R, Yamada A, Tsuchiya H, Kanamura K, Yamashita E, Kogure K. Protective effects of topical application of a poorly soluble antioxidant astaxanthin liposomal formulation on ultraviolet-induced skin damage. $J$. Pharm. Sci., 101, 2909-2916 (2012). 